\title{
Visual evoked potential changes in pre-diabetics, type 2 diabetics and normal subjects
}

\author{
Zaahid Naseer ${ }^{1}$, Arun Kumar M2,, Roopakala M S ${ }^{3}$, Pramila Kalra ${ }^{4}$ \\ ${ }^{1}$ Intern, ${ }^{2}$ Assistant Professor, ${ }^{3,4}$ Professor, ${ }^{1,2,4}$ Dept. of Physiology, ${ }^{3}$ Dept. of Endocrinology, Ramaiah Medical College, \\ Bangalore, Karnataka, India \\ *Corresponding Author: \\ Email: drarunkm@gmail.com
}

Received: $26^{\text {th }}$ January, 2018

Accepted: $26^{\text {th }}$ March, 2018

\begin{abstract}
Introduction: Diabetes mellitus type 2 is the most common metabolic disorder and diabetic retinopathy is the leading cause of blindness. Persistent hyperglycemia leads to damage of microvasculature supplying the retina and optic pathway. Visual evoked potential is the most commonly used test to evaluate the integrity of visual pathway. The purpose of the study is to compare the visual evoked potential latency and amplitude between the pre-diabetes, diabetes and normal controls.

Materials and Methods: There were three groups in the study with 23 patients in each group; pre-diabetic group, diabetic group and controls. Group was made based on fasting blood sugar levels. Pattern visual evoked potential was measured in all the groups by connecting scalp electrodes according to 10-20 International electrode placement system. Latency and amplitude of N75, P100 and N145 were measured and used for statistical analysis.

Results: All the parameters individually were compared between three groups using ANOVA test. There was no statistical difference between latencies and amplitude all the three parameters N75, P100, N145 in all the three channels O1-NE, O2- NE, Oz-NE in all the three groups.

Conclusion: There is no differences in the visual evoked potential latency and amplitude in the between pre-diabetics, diabetes and controls.
\end{abstract}

Keywords: Visual evoked Potential, Diabetic retinopathy, Pre-diabetes, P100, N75, N145.

\section{Introduction}

Diabetes mellitus (DM) is most common metabolic disorder in the world. Diabetes mellitus currently affects more than 62 million Indians, which is more than $7.1 \%$ of the adult population. ${ }^{1}$ It is a metabolic disorder which occurs due to defect in insulin secretion or insulin action or both. Lack of insulin leads to persistent hyperglycemia and other related complications. Chronic hyperglycemia is central to the pathophysiology of chronic complications such as cardiovascular and peripheral vascular disease, retinopathy, nephropathy, and neuropathy. ${ }^{2}$

Pre-diabetes is defined as elevated blood sugar that does not reach the criterion accepted for an outright diagnosis of diabetes. Pre-diabetics have an elevated risk for development of frank diabetes. Approximately 77 million adults are pre-diabetic in India. Diabetes is the leading cause of blindness between the ages of 20 and 74 across the globe. It is a serious problem because individuals with diabetes mellitus are 25 times more likely to get blindness. Blindness is primarily the result of progressive diabetic retinopathy and clinically significant macular edema. Duration of diabetes and degree of glycemic control are the best predictors of the development of retinopathy and subsequent optic nerve damage. $^{3}$

Visual evoked potential (VEP) is the commonly used neurophysiological test for evaluating the functions and integrity of the visual pathway. Clinically, the VEP is used in the testing of diseases related to the areas of refraction, infant acuity, diseases of the optic nerve, color blindness, amblyopia and field defects. ${ }^{4}$ Evoked potential are the tests wherein the stimulus of particular type are given and electrical response to the stimuli are recorded using appropriate instrument. In VEP the visual stimulus is given and responses are captured by placing electrodes on the occipital region. Two types of VEP are available based on the stimuli that are given. There are three types of standardized visual stimuli that are described in standards and guidelines for clinical electrophysiology of vision 2016 (ISCEV 2016). ${ }^{5}$ There are pattern reversal, pattern onset and flash. Pattern VEP and flash VEP wherein responses that are evoked by pattern stimuli and flash stimuli respectively. Pattern VEP testing is known to detect minor visual pathway abnormality with much greater sensitivity and accuracy. Checkerboard pattern reversal stimulation is the gold standard. ${ }^{6}$

VEP is described using different waves, its latency and amplitude. The most common wave that is used in the VEP is P100 (first positive wave) and other uncommon waves are N75and N145 (negative waves). These N75, P100 and N145 actually means the wave occur around $75 \mathrm{~ms}, 100 \mathrm{~ms}$ and $145 \mathrm{~ms}$ respectively with deviation on either side. The P100 waveform of VEP is generated in the visual areas occipital cortex and also thalamocortical volleys. ${ }^{7,8}$

There are studies that have described the changes of VEP in diabetes mellitus, and have found that there 
is impaired VEP latency. ${ }^{9}$ VEP studies have been done in several condition related to diabetes and its complications. ${ }^{10}$ There are no studies which have considered done comparing VEP latency changes between diabetic and pre-diabetic condition. There could be a possibility of optic nerve/pathway damage that could occur as a result of hyperglycemia in prediabetes. The aim of the study was to detect the latency and amplitude changes in pre-diabetics and diabetic patients and compare them with normal subjects.

\section{Materials and Methods}

The study was conducted at M S Ramaiah Medical College and Memorial Hospitals. It was a case control study with 23 subjects in each of three groups: prediabetic, diabetic and control group. The sample size was calculated based on the study conducted on the Visual evoked potential in non-insulin dependent diabetes retinopathy it was found that mean P100 latency of both eyes in diabetes patient was $110.14 \pm$ $5.30 \mathrm{~ms}($ mean $\pm \mathrm{SD}) .{ }^{1}$ In controls $100.17 \pm 0.75 \mathrm{~ms}$, so with a power of $95 \%$ and confidence level of $95 \%$ it was proposed to include 23 patients of diabetes, 23 subjects of pre-diabetes and 23 controls in present study with an effect size of 1.32 . The study was approved by institutional ethical committee.

The groups were made based on the fasting blood sugar (FBS) levels according to WHO Criteria. ${ }^{5}$ Fasting blood sugar level less than $100 \mathrm{gm} / \mathrm{dl}$, more than 125 $\mathrm{mg} / \mathrm{dl}$ are considered to be normal and diabetic group respectively. FBS levels between 100-125 mg/dl in considered in pre-diabetes group. Patients were in the age group of group was 30-60 years were included in the study. Alcoholics, smokers and patients with local eye disorders and abnormal fundoscopy were excluded from study. The FBS was determined by hexokinase method using the early morning samples collected from the after overnight fasting.

The study participants were explained about procedure of the visual evoked potential (VEP) and consent was obtained. If the patient had applied oil to head, they were asked to come on another day after taking head bath. If the participants were using spectacles they were asked to wear them during test procedure. The pattern VEP was measured and analyzed in all the groups by using Galileo NT instrument and software. The skin is prepared by abrading and degreasing. Five surface EEG electrodes were used and placed according to 10-20 international system. The recording electrodes were placed at $\mathrm{Oz}, \mathrm{O} 1$ and $\mathrm{O} 2$ using conducting jelly. $\mathrm{Oz}$ is a point $5 \mathrm{~cm}$ above the inion in the occipital region, $5 \mathrm{~cm}$ to the left of it $\mathrm{O} 1$ and $5 \mathrm{~cm}$ right to that is $\mathrm{O} 2$. The reference electrode (NE) is placed at Fpz or $12 \mathrm{~cm}$ above the nasion. The ground electrode is placed at the vertex, i.e. at $\mathrm{Cz}$. Resistance of less than 5 ohms was considered acceptable. Stimuli used is a standard checkerboard pattern which alternates at the frequency of $10 / \mathrm{sec}$ and is projected on the computer screen. Subjects were asked to focus at the center regions of screen with one eye at the time, the other eye was covered. Average of 200 responses was taken for interpreting the latencies and amplitudes. Latencies marked were N75, P100 and P145 by stimulus giving to both the eyes separately. Latency and amplitudes of first negative wave (N75), first positive wave (P100) and second negative wave (N145) was determined for all three leads i.e., O1-NE, $\mathrm{O} 2-\mathrm{NE}$ and OZ-NE.

Data was tabulated and analyzed using MS-Excel and SPSS software version 17. The statistical test used was descriptive analysis with mean and SD for baseline parameters. The latency and amplitude were compared using ANOVA analysis.

\section{Results}

The study consists of 23 subjects in each of 3 groups i.e., pre-diabetes group, diabetes group and control group. The mean and SD values of VEP latencies of N75, P100 and N145 in the three leads i.e., O1-NE, O2-NE and OZ-NE measured from right eye stimulus and left eye stimulus are depicted in table 1 and 2 respectively. Mean values of latencies of N75, P100 and N145 is more in diabetes group when compared to the pre-diabetic group which is more when compared to the control group. On application of one way analysis of variance (ANOVA) there was no significant difference in all the parameters.

Table 1: Comparison VEP latencies in the groups with right eye stimulus

\begin{tabular}{|c|c|c|c|c|c|}
\hline Channel & Latency & Prediabetic group & Diabetic group & Control group & P value \\
\hline O1-NE & N75 & $76.47 \pm 4.513$ & $75.93 \pm 6.772$ & $71.99 \pm 9.831$ & 0.107 \\
\hline & P100 & $103.59 \pm 4.703$ & $105.25 \pm 12.714$ & $100.16 \pm 11.791$ & 0.270 \\
\hline & N145 & $140.32 \pm 19.671$ & $154.98 \pm 37.361$ & $143.84 \pm 34.488$ & 0.314 \\
\hline O2-NE & N75 & $74.15 \pm 4.293$ & $76.74 \pm 9.921$ & $73.82 \pm 7.367$ & 0.401 \\
\hline & P100 & $103.86 \pm 6.198$ & $106.28 \pm 11.94$ & $100.04 \pm 20.699$ & 0.375 \\
\hline & N145 & $143.92 \pm 13.335$ & $157.02 \pm 38.337$ & $141.48 \pm 21.656$ & 0.133 \\
\hline Oz-NE & N75 & $76.83 \pm 7.591$ & $74.06 \pm 6.512$ & $73.1 \pm 7.171$ & 0.220 \\
\hline & P100 & $104.38 \pm 7.942$ & $101.77 \pm 4.338$ & $101.44 \pm 5.089$ & 0.229 \\
\hline & N145 & $144.41 \pm 15.716$ & $143.65 \pm 10.51$ & $142.96 \pm 20.419$ & 0.959 \\
\hline
\end{tabular}

$\mathrm{p}$ value $<0.05$ was considered statistical difference. 
Comparison of amplitudes of $\mathrm{P} 100$ in all the channels, O1-NE, O2- NE and Oz-NE was done with stimulus on right and left eye. The Mean values of amplitudes of P100 are almost same in the diabetes group when compared to the pre-diabetic group and control group. On application of one way analysis of variance (ANOVA) there was no significant difference in between the parameters in all the 3 groups. The values are mentioned in table 3 .

Table 2: Comparison VEP latencies in the groups with left eye stimulus

\begin{tabular}{|l|c|c|c|c|c|}
\hline Channel & Latency & Prediabetic group & Diabetic group & Control group & P value \\
\hline O1-NE & N75 & $74.77 \pm 5.989$ & $75.47 \pm 6.78$ & $72.88 \pm 7.194$ & 0.423 \\
\hline & P100 & $104.86 \pm 10.91$ & $103.17 \pm 8.819$ & $102.85 \pm 6.222$ & 0.729 \\
\hline & N145 & $144.86 \pm 9.133$ & $147.2 \pm 17.062$ & $138.24 \pm 14.803$ & 0.103 \\
\hline O2-NE & N75 & $75.04 \pm 9.056$ & $75.65 \pm 6.394$ & $74.02 \pm 8.646$ & 0.401 \\
\hline & P100 & $105 \pm 6.263$ & $109.84 \pm 13.436$ & $103.24 \pm 6.39$ & 0.375 \\
\hline & N145 & $149.07 \pm 38.996$ & $150.24 \pm 18.798$ & $142.06 \pm 15.84$ & 0.133 \\
\hline Oz-NE & N75 & $77.9 \pm 9.728$ & $76.01 \pm 8.764$ & $74.12 \pm 7.208$ & 0.358 \\
\hline & P100 & $104.4 \pm 7.951$ & $102.67 \pm 7.228$ & $100.52 \pm 6.403$ & 0.214 \\
\hline & N145 & $145.59 \pm 7.38$ & $146.84 \pm 19.278$ & $140.22 \pm 8.153$ & 0.192 \\
\hline
\end{tabular}

$\mathrm{p}$ value $<0.05$ was considered statistical difference.

Table 3: Comparison VEP amplitudes in all the groups with both eye stimulus

\begin{tabular}{|l|c|c|c|c|c|c|c|c|c|c|c|}
\hline Channel & $\begin{array}{c}\text { Stimulus } \\
\text { Side and } \\
\text { Wave }\end{array}$ & \multicolumn{3}{|c|}{$\begin{array}{c}\text { Control Group } \\
\text { (Mean } \pm \text { SD) }\end{array}$} & \multicolumn{2}{|c|}{$\begin{array}{c}\text { Prediabetic Group } \\
\text { (Mean } \pm \text { SD) }\end{array}$} & \multicolumn{2}{|c|}{$\begin{array}{c}\text { Diabetic Group } \\
\text { (Mean } \pm \text { SD) }\end{array}$} & p value \\
\hline O1-NE & Right P100 & 3.69 & \pm & 2.32 & 4.12 & \pm & 2.09 & 4.51 & \pm & 2.61 & 0.78 \\
\cline { 2 - 14 } & Left P100 & 3.38 & \pm & 1.89 & 4.53 & \pm & 2.02 & 5.12 & \pm & 3.1 & 0.378 \\
\hline O2-NE & Right P100 & 4.1 & \pm & 2.4 & 4.57 & \pm & 2.97 & 4.48 & \pm & 2.28 & 0.93 \\
\cline { 2 - 13 } & Left P100 & 3.89 & \pm & 2.42 & 4.27 & \pm & 2.68 & 4.79 & \pm & 2.36 & 0.755 \\
\hline Oz-NE & Right P100 & 2.23 & \pm & 3.4 & 4.94 & \pm & 5.55 & 6.55 & \pm & 2.78 & 0.115 \\
\cline { 2 - 11 } & Left P100 & 1.97 & \pm & 2.49 & 4.8 & \pm & 5.45 & 6.84 & \pm & 2.97 & 0.055 \\
\hline
\end{tabular}

$\mathrm{p}<0.05$ is considered significant

\section{Discussion}

Diabetes is the most common disease and its complication is life threatening. As the duration of diabetes increases and the occurrence of complications also increases. Most often patient reaches the hospital during the stage wherein the disease and its complication had made the patient morbid. Management of such conditions is difficult and challenging. It becomes important to detect such complications in the early stage given an indication to the patient about its impending danger. In this study an attempt was made to identify if it is possible to detect if there was any early damage to the optic nerve and its pathway in patients with Prediabetic condition.

Visual evoked potential is the one of the commonly used test in the assessment of the integrity of optic nerve pathway. When the stimulus is given the light gets processed from the retina enters the optic nerve, optic chaisma, and then enters in the visual area in the occipital area. Electrodes on the scalp is placed on the $\mathrm{O} 1$ and $\mathrm{O} 2$, these areas corresponds to the scalp region above left and right occipital cortex respectively whereas as $\mathrm{Oz}$ corresponds to the center of these to waves. ${ }^{11,12}$

In all the channels, i.e., O1-NE, O2-NE and OzNE, the N75, P100 and N145 did not show any significant change between the three groups. There is no significant delay in the latencies of these waves in the diabetic or prediabetic group. This is in contrary to the studies like Brian et al 2010 who has reported that there is increase in the latency in the subjects with diabetes. ${ }^{13}$ In another study conducted by Karlica ${ }^{14}$ et al it is had been reported that latencies of P100 is significantly delayed in diabetes mellitus type I subjects. There are other studies which have studied the effect the blood glucose on VEP recordings in the normal individuals and patient with diabetic retinopathy. ${ }^{15,16}$

Diabetes mellitus and its complication are better studied by understanding the pathophysiological process involved in development of such complications. Anticipation of complication in the pre-diabetic stage seems to be too early to get the overt effects of retina or optic nerve damage. However few studies are suggestive of increased macular choroidal thickness as the earliest determiner to detect the onset of diabetic retinopathy in pre-diabetes. ${ }^{17}$ In a cohort study, it was found that the prevalence of pre-diabetes was $22.4 \%$, and of diabetic retinopathy was $8.1 \%$ with majority of participants having mild Diabetic retinopathy (7.2\%). ${ }^{18}$ Diabetic retinopathy is classified into two stages: nonproliferative and proliferative. The pathophysiological mechanisms invoked in non-proliferative retinopathy 
include loss of retinal pericyctes, increased retinal vascular permeability, alterations in retinal blood flow, and abnormal retinal microvasculature, all of which lead to retinal ischemia. The appearance of neovascularization in response to retinal hypoxemia is hallmark of proliferative diabetic retinopathy. These newly formed vessels appear near the optic nerve and/or macula and rupture easily, leading to vitreous hemorrhage, fibrosis, and ultimately retinal detachment. ${ }^{19,20}$ On prediabetic condition there are several clinical observations recognizing that diabetesspecific microangiopathic complications such as diabetic retinopathy and others might be observed due to persistent hyperglycemia. ${ }^{21,22}$

Duration of diabetes, in an individual, is important factor which affects the latencies of VEP. As the person spends more time with uncontrolled status or increased blood sugar it has an adverse effect on the microvasculature of various systems. ${ }^{23}$ Circumference of the head is one of the major factors which could affect the latencies in visual evoked potential. It said that the length of optic nerve increases as the head circumference increases and correspondingly there is increase in the latency of these waves. These factors have been ignored in the study and add to the limitation of the study. ${ }^{24}$

It can be concluded that the there is significant changes in the latencies and amplitude of VEP measurements between diabetes, prediabetes and the study group. The future studies with larger sample size have to be considered to evaluate the possibility of using pattern VEP in the early detection of complications in diabetes.

Acknowledgement: The study was done as project for Indian Council for Medical Research- Short term studentship - 2014. (ICMR-STS-2014)

\section{References}

1. Kaivan Khavandi, Halima Amer, Bashar Ibrahim and Jack Brownrigg. Strategies for preventing type 2 diabetes: an update for clinicians. Ther Adv Chronic Dis. 2013;4(5): 242-261.

2. Loewen SL, Haas LB. Complications of diabetes: acute and chronic. Nurse Pract Forum. 1991 Sep;2(3):181-7.

3. Lamparter J, Raum P, Pfeiffer N, Peto T, Höhn R, Elflein $\mathrm{H}$, et al. Prevalence and associations of diabetic retinopathy large cohort of prediabetic subjects: the Gutenberg Health Study. J Diabetes Complicat. 2014 Aug;28(4):482-7.

4. Sharma R, Joshi S, Singh KD, Kumar A. Visual Evoked Potentials: Normative Values and Gender Differences. $J$ Clin Diagn Res. 2015 Jul;9(7):CC12-5.

5. Thompson DA, Fritsch DM, Hardy SE. The changing shape of the ISCEV standard pattern onset VEP. Doc Ophthalmol. 2017;135(1):69-76.

6. Fritsch DM, Sowden JC, Thompson DA. Pattern Onset ERGs and VEPs Produced by Patterns Arising From Light Increment and Decrement. Invest Ophthalmol Vis Sci. 2018 Jan 1;59(1):94-9.
7. Török B, Meyer M, Wildberger H. The influence of pattern size on amplitude, latency and wave form of retinal and cortical potentials elicited by checkerboard pattern reversal and stimulus onset-offset. Electroencephalogr Clin Neurophysiol. 1992 Feb;84(1):13-9.

8. Luigi Rovati, Giorgia Salvatori, Luca Bulf and Sergio Fonda. Optical and electrical recording of neural activity evoked by graded contrast visual stimulus. Bio Medical Engineering On Line 2007;6:28.

9. Heravian J, Ehyaei A, Shoeibi N, Azimi A, OstadiMoghaddam H, Yekta A-A, et al. Pattern Visual Evoked Potentials in Patients with Type II Diabetes Mellitus. $J$ Ophthalmic Vis Res. 2012 Jul;7(3):225-30.

10. Pescosolido N, Barbato A, Stefanucci A, Buomprisco G. Role of Electrophysiology in the Early Diagnosis and Follow-Up of Diabetic Retinopathy. J Diabetes Res. 2015;2015:319692.

11. Szabela DA, Loba J, Pałenga-Pydyn D, Tybor K, Ruxer J, Split W. [The picture of visual evoked potentials in type 2 diabetes mellitus]. Klin Oczna. 2005;107(7-9):498-501.

12. Luigi Rovati, Giorgia Salvatori, Luca Bulf and Sergio Fonda. Optical and electrical recording of neural activity evoked by graded contrast visual stimulus. Bio Medical Engineering On Line 2007;6:28.

13. Brian E. Wolff, Marcus A. Bearse Jr. Marilyn E. Schneck. Multifocal VEP (mfVEP) reveals abnormal neuronal delays in diabetes. Doc Ophthalmol. (2010) 121:189-196.

14. Karlica D, Galetović D, Ivanisević M, Skrabić V, Znaor L, Jurisić D. Visual evoked potential can be used to detect a prediabetic form of diabetic retinopathy in patients with diabetes mellitus type I. Coll Antropol. 2010 Jun;34(2):525-9.

15. Marilyn E. Schneck, Brad Fortune, Eugene Switkes, Michael Crognale, and Anthony J. Adams. Acute Effects of Blood Glucose on Chromatic Visually Evoked Potentials in Persons with Diabetes and in Normal Persons. Invest Ophthalmol Vis Sci. 1997;38:800-810.

16. Balta O, Sungur G, Yakin M, Unlu N, Balta OB, Ornek F. Pattern Visual Evoked Potential Changes in Diabetic Patients without Retinopathy. J Ophthalmol. 2017;2017:8597629.

17. Yazgan S, Arpaci D, Celik HU, Dogan M, Isık I. Macular Choroidal Thickness May Be the Earliest Determiner to Detect the Onset of Diabetic Retinopathy in Patients with Pre-diabetes: A Prospective and Comparative Study. Curr Eye Res. 2017 Jul;42(7):1039-47.

18. Xue Chen, Shi Song Rong, Qihua Xu, Fang Yao Tang, Yuan Liu, Hong Gu et al. Diabetes Mellitus and Risk of Age-Related Macular Degeneration: A Systematic Review and Meta-Analysis. PLOS ONE. 2014;9(9):e108196.

19. Nguyen TT, Wong TY. Retinal vascular changes and diabetic retinopathy. Curr Diab Rep. 2009 Aug;9(4):27783.

20. Cheung N, Rogers SL, Donaghue KC, Jenkins AJ, Tikellis G, Wong TY. Retinal arteriolar dilation predicts retinopathy in adolescents with type 1 diabetes. Diabetes Care. 2008 Sep;31(9):1842-6.

21. Putz Z, Kempler P, Jermendy G. [Diabetes-specific complications in pre-diabetes]. Orv Hetil. $2009 \mathrm{Nov}$ 22;150(47):2139-45.

22. Hanssen KF, Bangstad HJ, Brinchmann-Hansen O, DahlJørgensen K. Blood glucose control and diabetic microvascular complications: long-term effects of nearnormoglycaemia. Diabet Med. 1992 Oct;9(8):697-705. 
23. Roychowdhury S, Koozekanani DD, Parhi KK.

Automated detection of neovascularization for proliferative diabetic retinopathy screening. Conf Proc IEEE Eng Med Biol Soc. 2016 Aug;2016:1300-3.

24. Ovais Karnain Wadoo, Monika Kamra, Surjit Singh, Sangeeta Gupta, Shikha Baisakhiya. The Effect of Head Size on Visual Evoked Potential in Healthy Adults of North India. Int J Biol Med Res. 2013;4(4):3699-3703.

How to cite this article: Naseer Z, Arun KM, Roopakala MS, Kalra P. Visual evoked potential changes in pre-diabetics, type 2 diabetics and normal subjects. Ind J Clin Anat Physiol. 2018;5(3):326-330. 